\title{
Correction to: Entropy Stable Finite Volume Approximations for Ideal Magnetohydrodynamics
}

\author{
Dominik Derigs ${ }^{1}$ - Gregor J. Gassner ${ }^{2}$. \\ Stefanie Walch ${ }^{1}$. Andrew R. Winters ${ }^{2}$
}

Published online: 14 September 2018

(C) Deutsche Mathematiker-Vereinigung and Springer-Verlag GmbH Deutschland, ein Teil von Springer Nature 2018

\section{Correction to: Jahresber. Dtsch. Math.-Ver. https://doi.org/10.1365/s13291-018-0178-9}

When we published this article, the biographies of Gregor J Gassner and Stefanie Walch have been mixed up during the type-setting process. Sadly, this mistake remained unnoticed.

The correct biographies are:

Gregor Gassner is a professor in numerical analysis/scientific computing at the Mathematical Institute at the University of Cologne. Gregor obtained a diploma degree in aerospace engineering (2004) and a diploma degree in mathematics (2005) from the University of Stuttgart. In 2009 he received his doctoral degree at the Institute of Aerodynamics and Gasdynamics, Stuttgart. He got an offer for a Junior Professorship at TU Braunschweig in computational fluid dynamics, an offer for scientific computing at the TU Hamburg-Harburg and an offer from University of Cologne, where he joined in 2013. In 2016 he received an ERC starting grant on the topic of adaptive, robust and highly efficient numerical methods for the simulation of non-linear conservation laws.

Stefanie Walch is a full professor for theoretical astrophysics at the University of Cologne since 2013. After the diploma in physics in 2004, Stefanie received her doctoral degree in theoretical physics from the Ludwig-Maximilians-Universität

The online version of the original article can be found under https://doi.org/10.1365/s13291-018-0178-9.

A.R. Winters

awinters@math.uni-koeln.de

1 I. Physikalisches Institut, Universität zu Köln, Zülpicher Straße 77, 50937 Köln, Germany

2 Mathematisches Institut, Universität zu Köln, Weyertal 86-90, 50931 Köln, Germany 
München in 2008. Afterwards she has spent 3 years at Cardiff University in Wales, and approximately 2 years at the Max-Planck-Institute for Astrophysics in Garching as a postdoctoral researcher before being appointed at the University of Cologne. She specialises in the physics of the interstellar medium including star formation and stellar feedback in the form of ionizing radiation, stellar winds, and supernovae. The evolution of gas in galaxies is studied using massively parallel, three-dimensional magneto-hydrodynamics simulations with additional (self-)gravity, radiative transfer, and chemical networks to follow the formation and dissociation of molecules and to bridge the gap to observations in different tracers. Synthetic observations ranging from X-ray emission to $\mathrm{CO}$ and dust polarization maps are now used to put modern theories of star formation and feedback to the test. In 2015, she received an ERC Starting Grant on the topic of "The radiative interstellar medium".

The original article has been corrected. The publisher apologises for this mistake. 